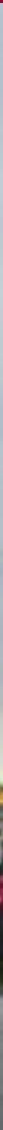

\title{
In-depth Analyse schwerer Unfälle mit schweren Lkw
}

Unfallforschung kompakt 

Inhalt

Einleitung

Sonderauswertung der amtlichen Unfallstatistik 5

Prospektive Unfallerhebung in Brandenburg 11

Schwere Lkw-Unfälle in der Unfalldatenbank der Versicherer 


\section{Einleitung}

Fast täglich berichten Medien über Unfälle mit Schwerverletzten oder gar Getöteten unter Beteiligung von Lkw, sei es beim Auffahren auf das Ende eines Staus auf Autobahnen oder bei innerstädtischen Unfällen in Kollisionen mit Fußgängern oder Radfahrern. Die Häufigkeit solcher Unfallereignisse und ihrer mitwirkenden Faktoren zu bestimmen und idealer Weise effektive Gegenmaßnahmen zu identifizieren, waren die Ziele des nachfolgend beschriebenen Forschungsprojekts der Unfallforschung der Versicherer (UDV) in Zusammenarbeit mit dem Lehrstuhl für Kraftfahrzeuge an der Technischen Universität Berlin.

Dabei lag der Schwerpunkt von Beginn an auf Ereignissen mit schweren Unfallfolgen, definiert als Unfälle mit Getöteten oder Schwerverletzten, und Beteiligung von schweren Güterkraftfahrzeugen. Deren Anzahl ist aus der öffentlich verfügbaren amtlichen Unfallstatistik nicht ohne weiteres zu ermitteln. Der Grund liegt im großen Spektrum der „Güterkraftfahrzeuge“, welches von Lieferwagen auf Pkw-Basis über Verteiler-Lkw bis zu Sattelzügen für den Fernverkehr und schweren Baustellenkippern reicht. Für die vorliegenden Forschungsfragen beschränkte sich die Betrachtung auf die vorrangig relevanten Fahrzeuge der EU-Typklasse „N3““. Dies sind definitionsgemäß Lkw mit einem zulässigen Gesamtgewicht über 12 Tonnen. Die Untergruppe „N3G“ kennzeichnet sogenannte geländegängige Fahrzeuge, die für den Betrieb abseits befestigter Straßen besonders geeignet sind. Innerhalb der Typklasse N3 sind verschiedene Bauarten möglich: Zwei-, drei- oder in Ausnahmefällen auch vierachsige Lkw, die ohne oder mit einem Anhänger eingesetzt werden können, und - in der Regel zweiachsige Sattelzugmaschinen, die einen Sattelauflieger ziehen. Das zulässige Gesamtgewicht des Gespanns darf - von Sondertransporten abgesehen - in Deutschland 40 Tonnen nicht überschreiten. 
Sonderauswertung der amtlichen Unfallstatistik

Das Forschungsprojekt stützt sich hauptsächlich auf drei Säulen: Im ersten Schritt auf eine Sonderauswertung der amtlichen Statistik schwerer Unfälle mit N3-Güterkraftfahrzeug-Beteiligung des Jahres 2014, auf eine Vollerhebung solcher Unfälle im Bundesland Brandenburg im Jahr 2016 sowie eine Analyse von 339 Unfällen mit schweren Lkw und Personenschaden der Unfallforschung der Versicherer. Damit ergibt sich ein umfassendes Bild von schweren Unfällen mit schweren Lkw, welches die Identifizierung charakteristischer Merkmale und Ansatzpunkte für die Prävention sowie die Einordnung im allgemeinen Unfallgeschehen in Deutschland ermöglicht.

\section{Sonderauswertung der amtlichen Unfallstatistik}

Rahmenbedingungen

Seit 2008 hat sich der Bestand an Güterkraftfahrzeugen in Deutschland um 16 Prozent erhöht. Dabei wird dieser Zuwachs in erster Linie von Kleintransportern getragen.
Die Anzahl von Lkw über 12 Tonnen zulässigem Gesamtgewicht, die entweder als Solo-Lkw oder mit einem Anhänger unterwegs sein können, stagnierte, aber die der Sattelzugmaschinen wuchs um knapp 5 Prozent. Wenngleich Sattelzugmaschinen mit 188.481 Einheiten im Jahr 2015 nur 7 Prozent an allen 2,7 Millionen in Deutschland zugelassenen Lkw aller Gewichtsklassen ausmachten, so trugen sie ein Viertel zur Gesamtfahrleistung aller Güterkraftfahrzeuge bei. Die durchschnittliche Fahrleistung einer Sattelzugmaschine wuchs von 2003 bis 2014 um ein Fünftel und übersteigt mit derzeit $103.000 \mathrm{~km}$ im Jahr die anderer Lkw-Arten und die von Pkw bei weitem. Das Verkehrsaufkommen (die transportierte Gütermenge in Tonnen) und die Verkehrsleistung (die transportierte Gütermenge multipliziert mit ihrer Transportweite in Kilometer) stiegen ebenfalls, insbesondere bei ausländischen Güterkraftfahrzeugen auf deutschen Straßen (Abb. 1). Bezogen auf das Referenzjahr 2003 nahmen bei diesen die Verkehrsleistung um 37 Prozent und das Verkehrsaufkommen um 45 Prozent zu.
- Abb. 1: Entwicklung von Verkehrsaufkommen und -leistung von Güterkraftfahrzeugen in Deutschland (Quelle: DIW Deutsches Institut für Wirtschaftsforschung)

Entwicklung von Verkehrsaufkommen (VA) und Verkehrsleistung (VL) von Güterkraftfahrzeugen in Deutschland

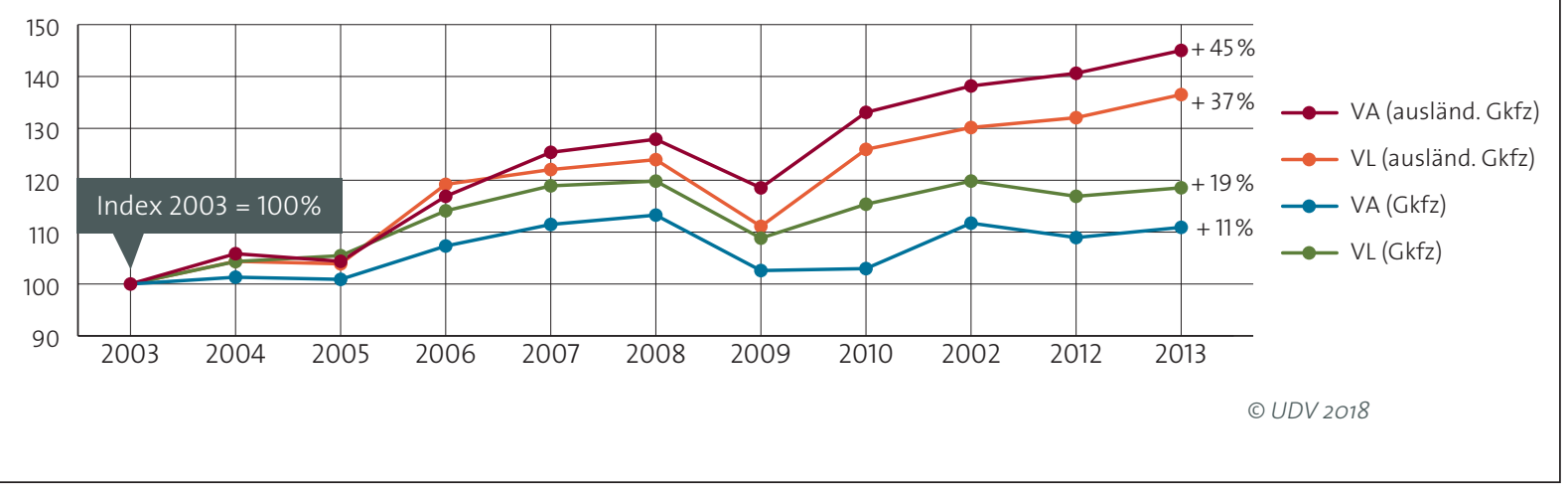




\section{Unfallentwicklung}

Bezogen auf die Fahrleistung sind N3-Sattelzugmaschinen seltener als Kraftfahrzeuge insgesamt, aber auch seltener als inländische Güterkraftfahrzeuge insgesamt in Unfälle mit Personenschaden verwickelt (Abb. 2). Werden jedoch schwere Unfälle mit Getöteten oder Schwerverletzten betrachtet, so übersteigt der Anteil mit 30 Prozent unter Sattelzugmaschinen den der Vergleichsgruppen mit 21 beziehungsweise 22 Prozent. Daraus lässt sich ablesen, dass die Unfallbeteiligung schwerer Lkw zwar unterdurchschnittlich ist, die Unfallfolgen dann aber oftmals schwerer ausfallen als im Unfallgeschehen anderer Kraftfahrzeuge.

Unfälle mit Personenschaden vs. Unfälle mit Schwerverletzten und Getöteten je 1 Mrd. km

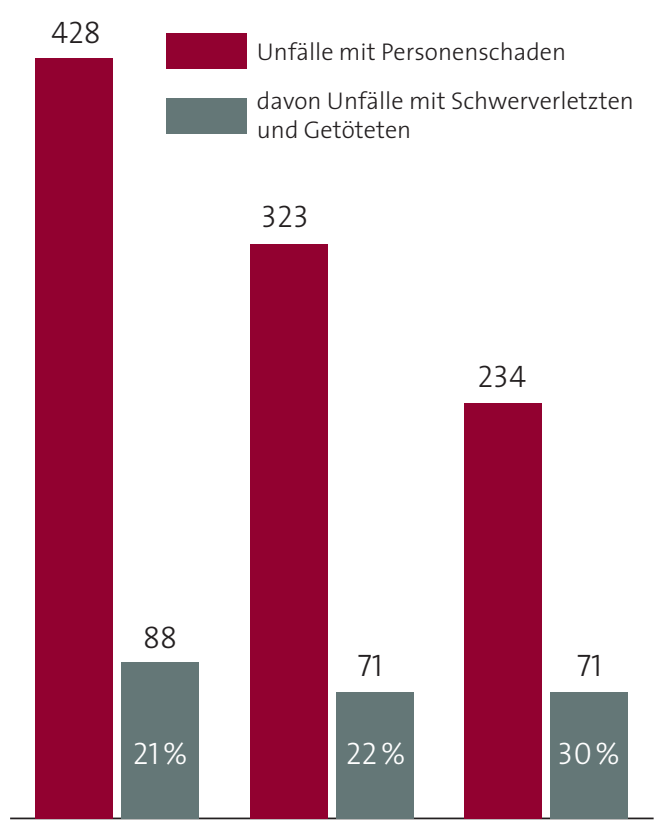

Kfz gesamt inländisches Gkfz N3 SZM
Seit 2005 hat die Anzahl Verunglückter bei Unfallbeteiligung von Güterkraftfahrzeugen eine Entwicklung genommen, die noch positiver verläuft als im allgemeinen Unfallgeschehen, und dies vor dem Hintergrund eines deutlich gestiegenen Verkehrs- und Gütertransportaufkommens. Zu befriedigen vermag sie dennoch nicht. Insgesamt starben 2014 bei Unfällen von Güterkraftfahrzeugen aller Art 759 Menschen (35 Prozent weniger als 2005) und 7.234 wurden schwer verletzt (18 Prozent weniger als 2005). Im amtlichen Sinne sind „Schwerverletzte“ Verunglückte, die für mindestens 24 Stunden stationär in einer Klinik aufgenommen werden und nicht innerhalb von 30 Tagen nach dem Unfallereignis versterben.

Für die weiteren Betrachtungen der Unfallbeteiligung von N3-Lkw lohnt es sich verschiedentlich, eine Unterscheidung zu treffen zwischen N3-Lkw ohne Anhänger und mit Anhänger und N3-Sattelzugmaschinen. Während sich N3-Lkw ohne Anhänger im regionalen Verteilerverkehr, aber auch als Bau- oder Entsorgungsfahrzeuge häufig innerorts bewegen, werden Sattelzugmaschinen beziehungsweise Sattelzüge vorzugsweise im Güterfern- und internationalen Verkehr eingesetzt und sind daher vorrangig auf Autobahnen unterwegs. Bei im Ausland zugelassenen Güterkraftfahrzeugen, die auf deutschen Straßen verunglücken, ist die Identifizierung von N3-Lkw in der amtlichen Statistik nicht möglich. Bei Sattelzugmaschinen kann aber davon ausgegangen werden, dass diese in aller Regel zur N3-Typklasse gehören. Ausländische Sattelzugmaschinen wurden bei der Sonderauswertung daher mit berücksichtigt, andere Lkw mit ausländischer Zulassung hingegen nicht.

Abb. 2:

Häufigkeit von Unfällen mit Personenschaden und schweren Unfällen (U(GT+SV)) bei Beteiligung von Kraftfahrzeugen allgemein, inländischen Güterkraftfahrzeugen und N3-Sattelzugmaschinen, bezogen auf 1 Milliarde zurückgelegte Kilometer 


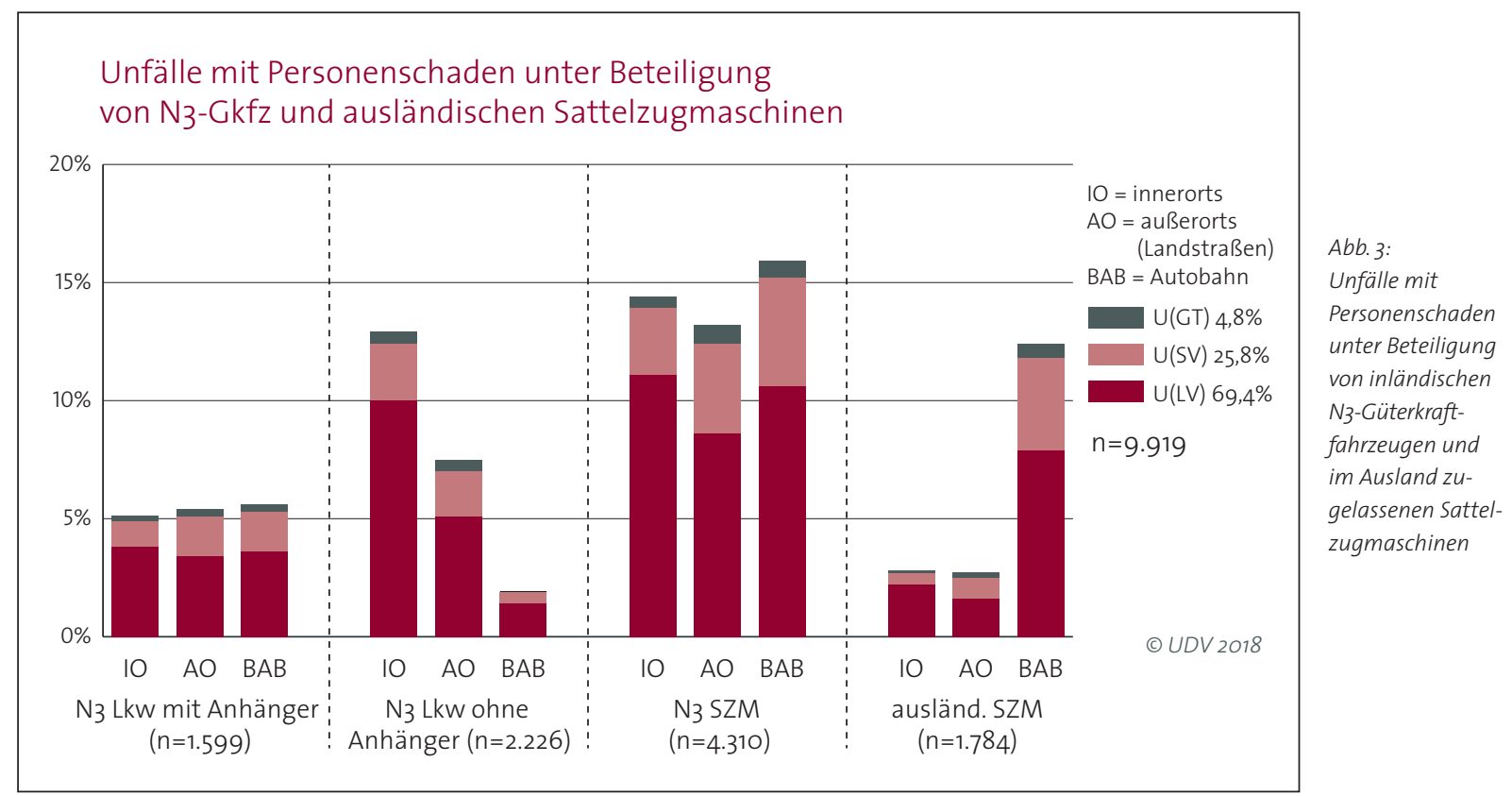

Aus Abb. 3 geht einerseits hervor, dass unter den N3-Güterkraftfahrzeugen, die 2014 in 9.919 Unfälle mit Personenschaden verwickelt waren, Sattelzugmaschinen mit 4.310 Unfällen die größte Gruppe stellen, gefolgt von N3Lkw ohne Anhänger. Andererseits verdeutlicht Abb. 3 auch die grundsätzlichen Unterschiede in der Ortslage der Unfälle abhängig von der Art des N3-Güterkraftfahrzeugs. Während Lkw mit Anhänger und inländische Sattelzugmaschinen zu jeweils etwa gleichen Teilen innerorts, außerorts auf Landstraßen und auf Autobahnen verunglücken, liegt der Schwerpunkt des Unfallgeschehens mit Beteiligung von N3-Lkw ohne Anhänger im Innerorts-Bereich. Auf Autobahnen verunfallen diese selten. Ausländische Sattelzugmaschinen wiederum verunglücken zum Großteil auf Autobahnen und nur selten auf Innerortsoder Außerortsstraßen. Etwa ein Viertel der Unfälle aller N3-Güterkraftfahrzeuge mit Personenschaden waren Unfälle mit Schwerverletzten $(U(S V))$; das heißt, als schwerste Unfallfolge waren ein oder mehrere Schwerverletzte, eventuell aber zusätzlich auch Leichtverletzte zu beklagen. Etwa jedes zwanzigste Ereignis war ein Unfall mit Getöteten $(U(G T))$, also mit einem oder mehreren Getöteten als schwerster Unfallfolge, möglicherweise aber auch mit zusätzlichen Schwer- oder Leichtverletzten.
Die verunglückten Verkehrsteilnehmer sind zu einem vergleichsweise geringen Anteil Insassen von N3-Güterkraftfahrzeugen: von 3.248 Schwerverletzten bei Unfällen mit N3-Beteiligung waren 560 (17 Prozent) N3-Fahrzeugführer oder deren Mitfahrer. Unter den 499 getöteten Personen in Unfällen mit N3-Güterkraftfahrzeugen starben 49 N3-Insassen (10 Prozent).

Alleinunfälle, also Unfälle ohne Beteiligung eines anderen Verkehrsteilnehmers, sind sowohl bei Güterkraftfahrzeugen im Allgemeinen als auch bei N3-Güterkraftfahrzeugen selten. In lediglich 6 Prozent der Unfälle mit Personenschaden verunglückt der Lkw ohne Beteiligung Dritter, bei Unfällen von Pkw liegt dieser Anteil bei 11 Prozent.

Dementsprechend sind N3-Güterkraftfahrzeuge größtenteils in Unfälle mit mindestens zwei Beteiligten verwickelt. Hauptverursacher des Unfalls, wie er von der Polizei anhand des Unfallhergangs festgestellt wird, sind die Führer des N3-Fahrzeugs (inländische N3-Fahrzeuge und ausländische Sattelzugmaschinen) in 51 Prozent der Fälle mit Personenschaden. Im Vergleich dazu sind Führer von Güterkraftfahrzeugen bis 12 Tonnen zulässigem Gesamtgewicht (Typklassen N1 und N2) und Führer der übrigen 
ausländischen Güterkraftfahrzeuge in zwei Drittel der Unfälle Hauptverursacher. Bei Unfällen von Pkw ist dessen Führer in 56 Prozent der Hauptverursacher. N3-Lkw beziehungsweise deren Fahrer sind also eher unterdurchschnittlich oft Unfallverursacher.

Unfallgegner

Bei Unfällen mit genau zwei Beteiligten lässt sich aus der amtlichen Unfallstatistik auch der Unfallgegner des schweren Lkw bestimmen. Häufigster Unfallgegner für N3-Güterkraftfahrzeuge ist demnach der Pkw mit 64 Prozent, gefolgt von anderen Güterkraftfahrzeugen und ungeschützten Verkehrsteilnehmern, den Fußgängern und Radfahrern, mit 14 bzw. 13 Prozent (Abb.4). Abhängig von der Art des Lkw, der Ortslage und der betrachteten Unfallschwere schwanken die Anteilswerte allerdings erheblich: zwischen 51 Prozent der Innerortsunfälle von N3-Lkw ohne Anhänger und 73 Prozent der Außerortsunfälle von N3-Sattelzugmaschinen auf Landstraßen haben einen Pkw als Unfallgegner, wenn Unfälle mit Personenschaden $(U(P))$ betrachtet werden. Beschränkt man die Betrachtung auf Unfälle mit Schwerverletzten (U(SV)), so bilden für N3-Sattelzugmaschinen andere Güterkraftfahrzeuge die zweitgrößte Gruppe von Unfallgegnern (18 Prozent), insbesondere auf Autobahnen. Bei Unfällen mit Schwerverletzten (U(SV)) und Getöteten $(U(G T))$ von N3-Lkw ohne Anhänger gehören Fußgänger und Radfahrer zu den häufigsten Unfallgegnern (37 Prozent), insbesondere innerorts. Die Häufigkeitsverteilung der Unfallgegner in Abhängigkeit von der Unfallschwere und der Ortslage spiegelt dabei die unterschiedlichen Einsatzarten wider, wie schwere Lkw eingesetzt werden: Lkw ohne Anhänger sind besonders im städtischen und regionalen Verteiler- und Baustellenverkehr unterwegs und daher besonders häufig in Unfälle mit Fußgängern und Radfahrern verwickelt. Sattelzüge dienen in erster Linie dem Gütertransport über große Entfernungen und verunfallen besonders häufig auf Landstraßen und Autobahnen, wobei dann auch andere Güterkraftfahrzeuge als Unfallgegner von Bedeutung sind.
Vergleich der Unfallgegner von Pkw und N3-Gkfz bei Unfällen mit Personenschaden mit zwei Beteiligten

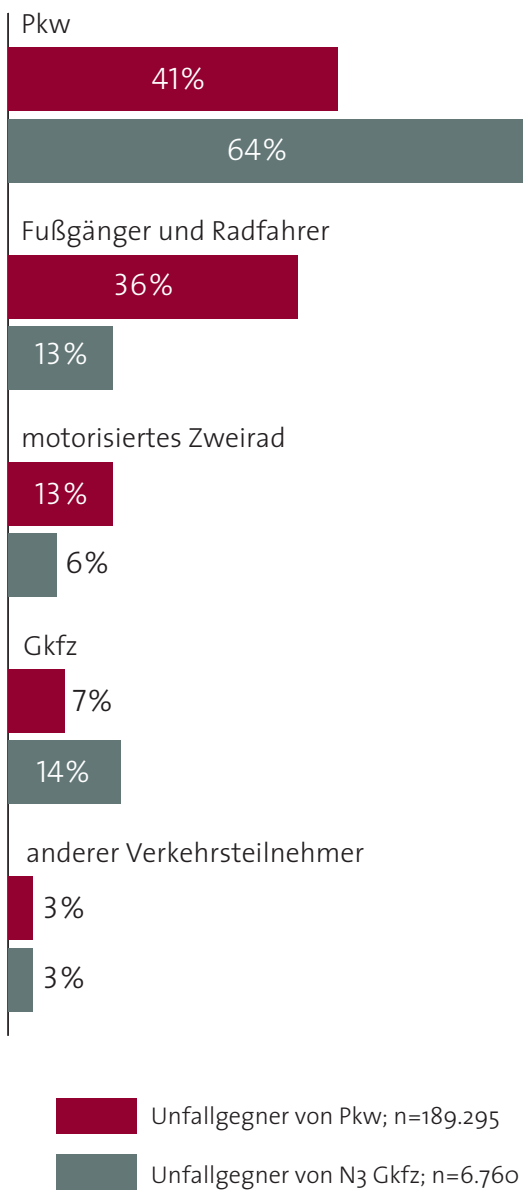

(c) UDV 2018

Abb. 4: Vergleich der Unfallgegner von N3-Güterkraftfahrzeugen und von Pkw bei Unfällen mit Personenschaden und genau zwei Beteiligten 
Sonderauswertung der amtlichen Unfallstatistik

\section{Unfallumstände}

Bei der Frage nach den Unfallursachen ist zunächst ein Blick auf den Unfallhergang hilfreich. In der amtlichen Verkehrsunfallstatistik eignen sich dafür der Unfalltyp, welcher die Konfliktsituation beschreibt, die dem Unfall unmittelbar vorausging, und die Unfallart, welche den ersten Kontakt zwischen Unfallbeteiligten oder mit Hindernissen beschreibt. Kombiniert man diese beiden Merkmale, so zeigen sich für Unfälle zwischen zwei Beteiligten mit Schwerverletzten und Getöteten, wenn der N3-Fahrer Hauptverursacher ist, einige wenige Schwerpunkte (Abb. 5).
Mit 18 Prozent haben Unfälle im Längsverkehr, bei denen es zum Zusammenstoß mit einem anderen Fahrzeug kommt, das vorausfährt oder wartet, den größten Anteil. Hierunter fällt das Auffahren eines N3-Lkw auf ein anderes Fahrzeug, aber auch umgekehrt das Auffahren eines anderen Verkehrsteilnehmers auf das Heck eines N3-Fahrzeugs. Unfälle im Längsverkehr und Zusammenstöße mit einem anderen Fahrzeug, das sich in gleicher Richtung bewegt, rangieren mit knapp 11 Prozent auf dem dritten Platz. Dies schließt beispielsweise Unfälle beim Spurwechsel ein. Weitere Schwerpunkte unter den Unfallhergängen sind Unfälle zwischen einem N3-Lkw und einem anderen Fahrzeug beim Einbiegen/Kreuzen

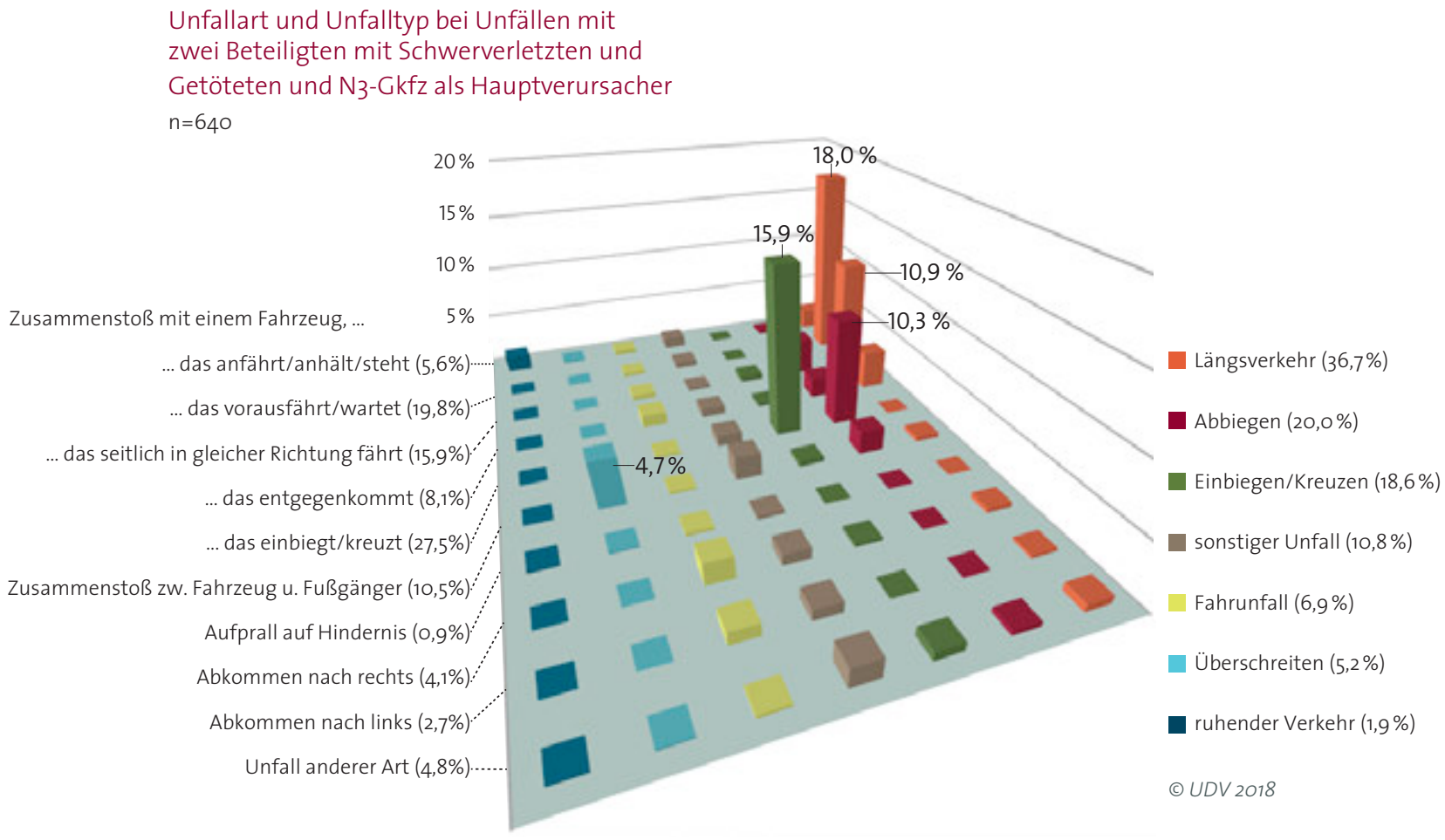

Abb. 5: Unfallart und Unfalltyp bei schweren Unfällen mit zwei Beteiligten von N3-Güterkraftfahrzeugen als Hauptverursacher 
Gegenüberstellung der Verkehrsstärke und der Anzahl der U(P) für Sattelzugmaschinen auf Bundesautobahnen

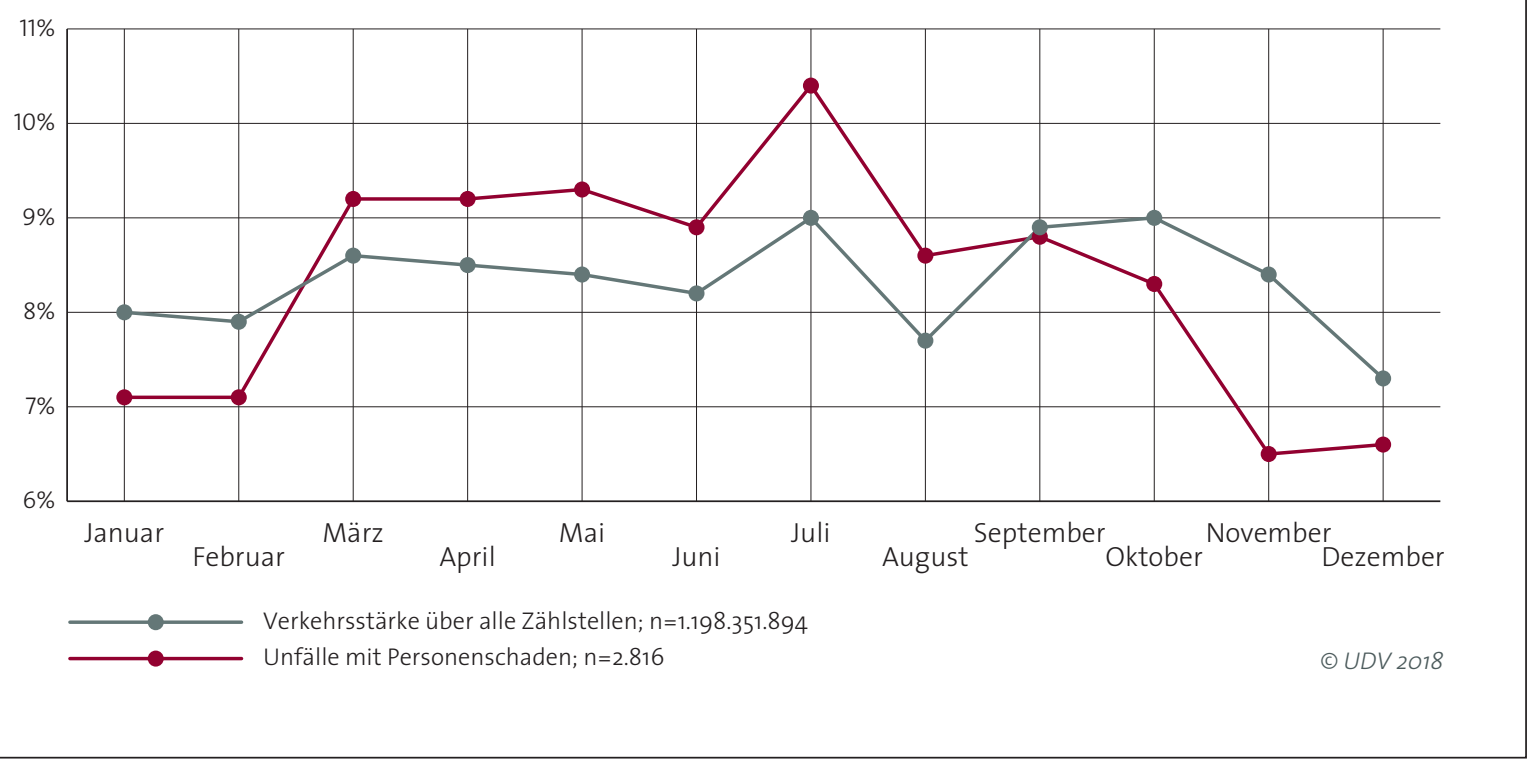

Abb. 6: Gegenüberstellung der Verteilungen

von Verkehrsstärke und Unfällen mit

Personenschaden für Sattelzugmaschinen

auf Bundesautobahnen

mit knapp 16 Prozent und beim Abbiegen mit gut 10 Prozent. Hierunter fallen unter anderem auch Kollisionen zwischen einem abbiegenden Lkw und einem Radfahrer. Der Unfalltyp „Überschreiten-Unfall“ ist allein Unfällen mit Fußgängern vorbehalten und stellt ein weiteres, wenngleich selteneres Szenario dar. Auffällig ist, dass Fahrunfälle - ein Unfalltyp, bei dem seitens des Hauptverursachers der Verlust der Kontrolle über das Fahrzeug als unfallauslösend zu vermuten ist - bei N3-Güterkraftfahrzeugen mit einen Anteil von knapp sieben Prozent selten zu finden ist. Alleinunfälle von N3-Güterkraftfahrzeugen sind - da definitionsgemäß nur ein Beteiligter vorhanden ist - in Abbildung 5 nicht dargestellt. 2014 starben zwar nur vier Insassen von N3-Fahrzeugen bei Alleinunfällen, aber 220 wurden schwer verletzt. Letztere repräsentieren 39 Prozent der 560 schwerverletzten N3-Insassen (einschließlich ausländischer Sattelzugmaschinen) des Jahres 2014.
Weitere Merkmale, die im Rahmen der Sonderauswertung der amtlichen Statistik untersucht wurden, wie Witterung, Jahres- und Tageszeit zeigen allgemein wenig Auffälligkeiten. Im jahreszeitlichen Verlauf ist bei Güterkraftfahrzeugen ähnlich wie bei Pkw eine Zunahme der Unfallzahlen in den Sommermonaten zu beobachten und ein Minimum in den Wintermonaten. Zum Teil lassen sich diese Anstiege und Abfälle bei N3-Güterkraftfahrzeugen im Zusammenhang mit der ebenfalls saisonal steigenden und fallenden Verkehrsstärke sehen. Allerdings fällt bei der gesonderten Betrachtung von Sattelzugmaschinen auf Autobahnen auf, dass von Mai bis August eine - verglichen mit der Verkehrsstärke dieser Fahrzeuge - deutlich überdurchschnittliche Unfallhäufigkeit herrscht, während diese von November bis Februar deutlich unterdurchschnittlich ist (Abb. 6). 
Prospektive Unfallerhebung in Brandenburg

Weil an Sonn- und Feiertagen allgemein ein Lkw-Fahrverbot gilt, sind an diesen Tagen - aber auch in Monaten, in denen kalendarisch bedingt besonders viele Fahrverbotstage liegen - N3-Unfälle naturgemäß wesentlich seltener. Im tageszeitlichen Verlauf liegen die Spitzen in den frühen Nachmittagsstunden von 12 bis 15 Uhr und den späten Vormittagsstunden von 9 bis 12 Uhr. Erwartungsgemäß findet ein Großteil der Unfälle von N3-Lkw bei trockenen Fahrbahnverhältnissen und bei Tageslicht statt.

Über die Jahre merklich gewandelt hat sich das Bild bei der Unfallbeteiligung ausländischer Lkw, veranschaulicht an Sattelzugmaschinen, die den Großteil der im internationalen Güterfernverkehr eingesetzten N3-Fahrzeuge ausmachen. Im Jahr 2001 waren diese noch an 1.026 Unfällen mit Personenschaden auf Autobahnen beteiligt und wurden hinsichtlich des Zulassungslandes von den Niederlanden, Polen, Österreich und der Tschechischen Republik angeführt. 2014 war die Anzahl der Personenschadenunfälle auf Autobahnen um ein Drittel auf 1.365 gestiegen, wobei die Rangfolge eindeutig von in Polen zugelassenen Sattelzugmaschinen angeführt wurde, gefolgt von der Tschechischen Republik, Rumänien und den Niederlanden.

\section{Prospektive Unfallerhebung in Brandenburg}

Erhebungsmethode

Während die Sonderauswertung der amtlichen Statistik wichtige Zahlen zum Unfallgeschehen mit Beteiligung von N3-Güterkraftfahrzeugen - zumindest für in Deutschland zugelassene Lkw - auf Bundesebene liefert, so lässt sie doch noch wichtige Fragen offen. Beispielsweise wurde erkannt, dass Unfälle im Längsverkehr mit Auffahren auf Vorausfahrende oder Wartende einen der Schwerpunkte im Unfallgeschehen mit Schwerverletzten und Getöteten repräsentieren. Nicht beantwortet ist damit jedoch die Frage, wie häufig ein N3-Lkw auf ein anderes Fahrzeug auffährt und umgekehrt, wie oft ein anderer Verkehrsteilnehmer auf ein N3-Fahrzeug auffährt. Zur Beantwortung solcher Fragestellungen wurden 2016 alle Unfälle im Bundesland Brandenburg mit Beteiligung von N3-Güterkraftfahrzeugen und schweren Unfallfolgen (mindestens eine schwerverletzte oder getötete Person) erhoben und ausgewertet. Dies geschah mit freundlicher Unterstützung des Polizeipräsidiums Brandenburg, welches anonymisierte Daten aus den betreffenden Verkehrsunfallanzeigen zur Verfügung stellte. Ergänzt wurde diese Vollerhebung durch zeitnahe Internetrecherchen zu den in Frage kommenden Unfallereignissen. Dadurch konnten Sachverhalte, wie beispielsweise die Zugehörigkeit eines verunfallten Lkw zur interessierenden N3-Typklasse, verifiziert werden. So gelang auch die Einordnung ausländischer Lkw anhand der Typklassen, was mit der amtlichen Statistik nur eingeschränkt möglich war. Zu berücksichtigen ist, dass Brandenburg als Bundesland mit vorwiegend ländlicher Prägung nicht ohne weiteres als repräsentativ für das Unfallgeschehen mit schweren Lkw in der Bundesrepublik angesehen werden kann. Mit seinem überdurchschnittlich hohen Autobahnanteil am Straßennetz und starkem Transitverkehr gerade aus und in die östlichen Staaten ist es aber prädestiniert dafür, Unfälle mit N3-Güterkraftfahrzeugen auf Außerortsstraßen und besonders Autobahnen zu untersuchen (Abb. 7). 


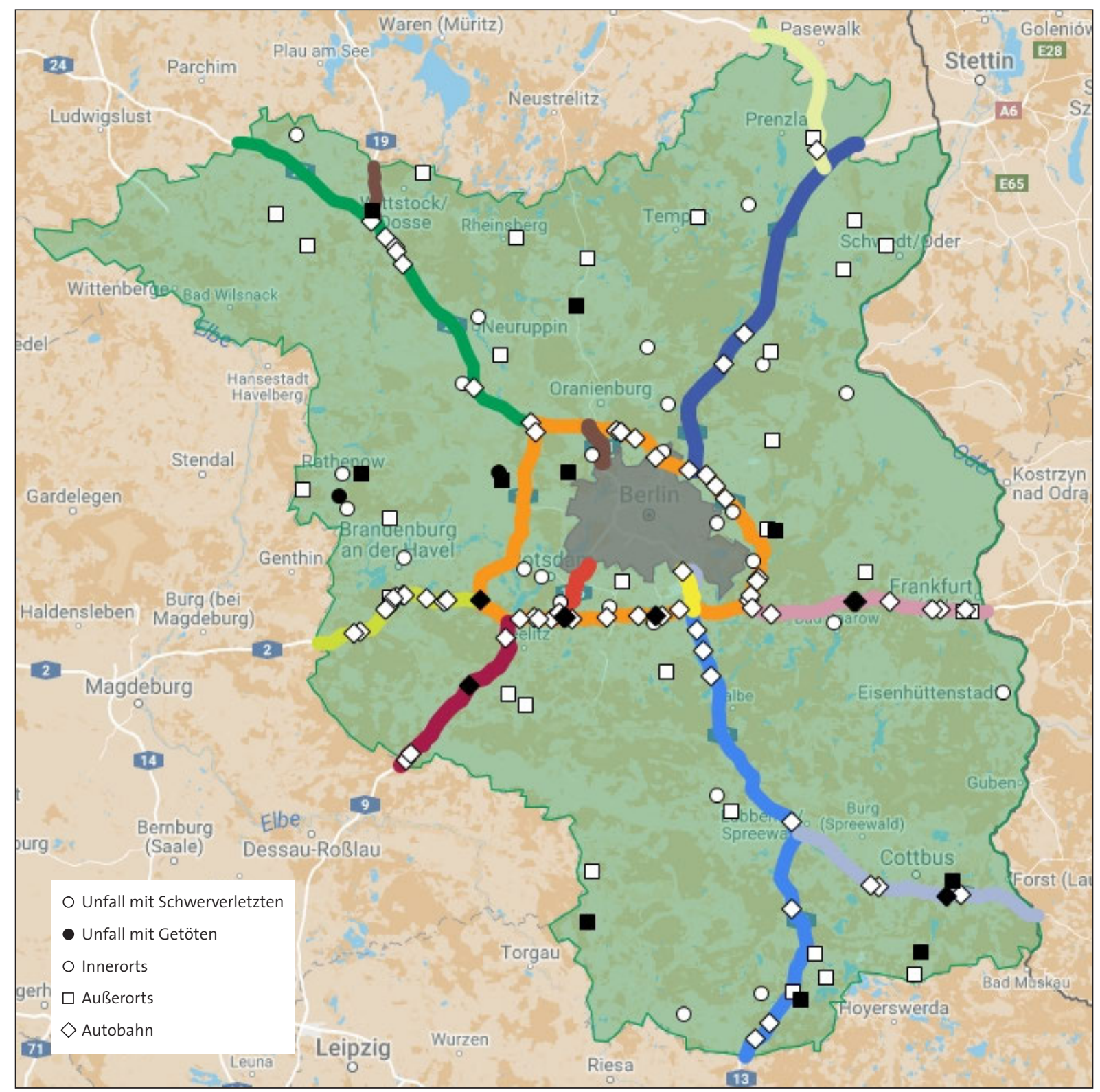

Abb. 7: Geographische Lage der Unfälle mit Schwerverletzten und Getöteten unter Beteiligung von N3-Güterkraftfahrzeugen in Brandenburg im Jahr 2016 (Kartendaten: Google, GeoBasis-DE/BKG) 
Tatsächlich bestätigt der Vergleich mit dem schweren Unfallgeschehen mit N3-Beteiligung im Bundesgebiet 2014, dass in Brandenburg der Anteil von Innerorts- und Außerorts-Unfällen etwas geringer, der von Autobahnunfällen hingegen deutlich höher als im Bundesdurchschnitt ausfällt (Abb. 8).

Schwerverletzte und Getötete bei schweren Unfällen unter Beteiligung von N3-Güterkraftfahrzeugen nach Ortslage

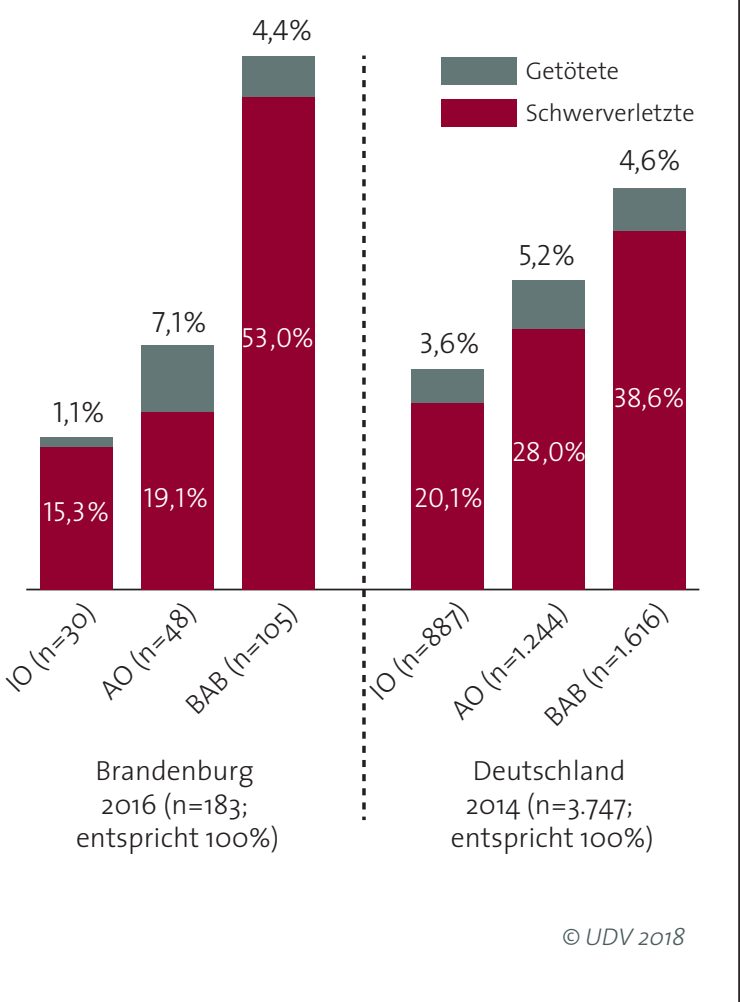

Abb. 8: Schwerverletzte und Getötete bei schweren Unfällen mit Beteiligung von N3-Güterkraftfahrzeugen nach Ortslage in Brandenburg 2016 und in Deutschland 2014 
Unfallgeschehen im Land Brandenburg

Im Jahr 2016 ereigneten sich in Brandenburg 145 Unfälle mit Beteiligung von N3-Güterkraftfahrzeugen und mit schweren Unfallfolgen $(\mathrm{U}(\mathrm{GT})$ und $\mathrm{U}(\mathrm{SV}))$ ). Darin waren insgesamt 319 Beteiligte verwickelt, darunter 173 N3-Güterkraftfahrzeuge (Abb. 9). In diese 145 Unfallereignisse waren insgesamt 407 Fahrzeugnutzer und Fußgänger involviert, von denen 23 getötet, 160 schwer und 52 leicht verletzt wurden. Davon waren sechs getötete und 43 schwer verletzte Insassen von N3-Güterkraftfahrzeugen.
Dabei bestätigt sich die Erkenntnis aus der Sonderauswertung der Bundesstatistik, dass sich schwere Unfälle von Sattelzugmaschinen vorrangig auf Autobahnen und die von N3-Lkw ohne Anhänger meist innerorts ereignen. Mit 13 schwerverletzten Radfahrern und einem getöteten Fußgänger waren schwere Unfälle zwischen N3-Lkw und ungeschützten Verkehrsteilnehmern im Vergleich zum bundesweiten Unfallgeschehen seltener. Dementsprechend verwundert es nicht, dass sich die Verteilung der Unfalltypen und Unfallarten in Brandenburg zwar ähnlich wie im Bundesdurchschnitt darstellt, Einbiege- und Abbiegeunfälle innerorts aber eine geringere Bedeutung haben zugunsten eines gestiegenen Anteils von Unfällen im Längsverkehr auf Landstraßen und Autobahnen.

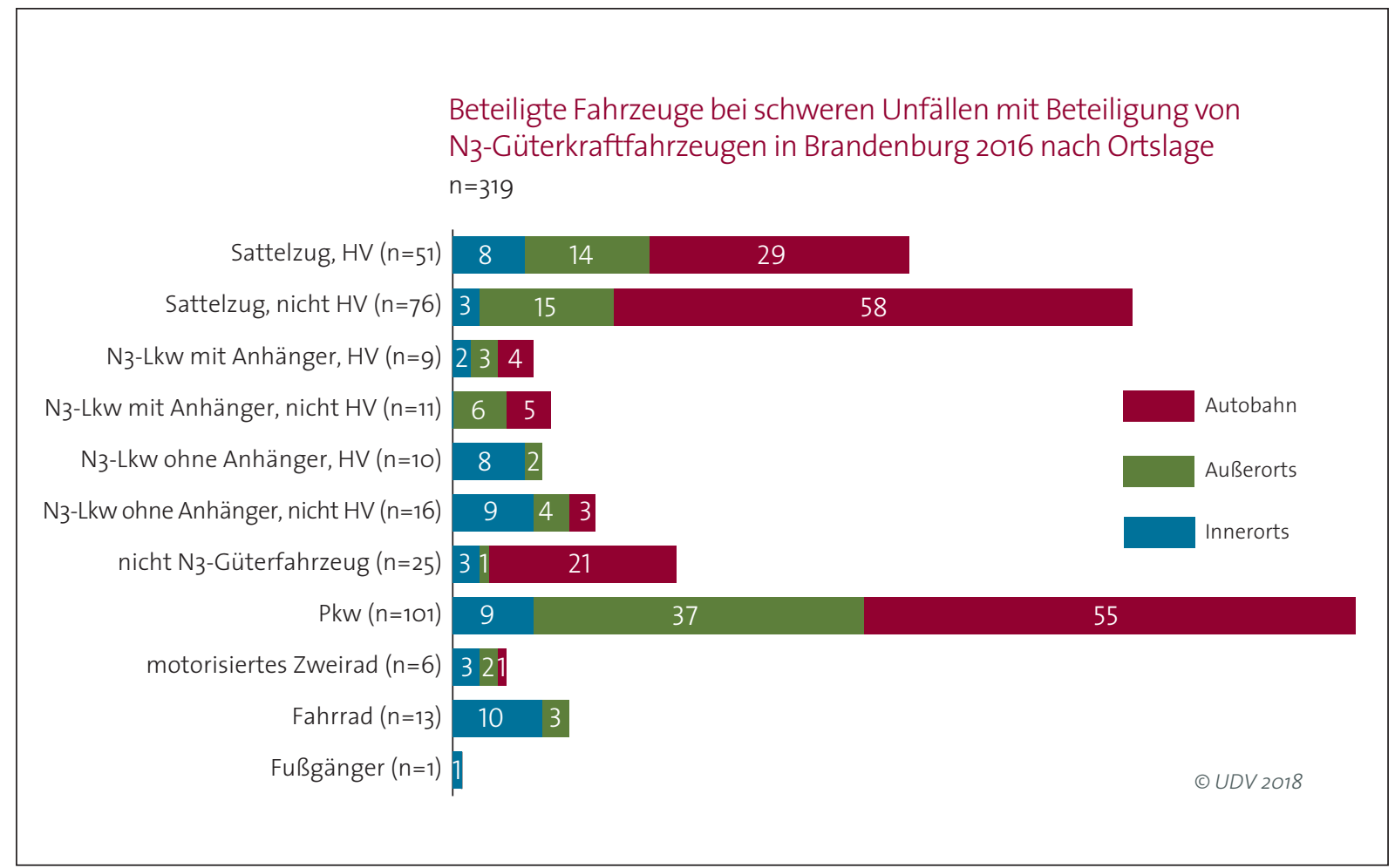

Abb. 9: Art der Verkehrsbeteiligung bei Unfällen mit Schwerverletzten und Getöteten unter Beteiligung von N3-Güterkraftfahrzeugen einschließlich Alleinunfällen in Brandenburg im Jahr 2016 
Prospektive Unfallerhebung in Brandenburg

Mit 20 Ereignissen, welche 14 Prozent entsprechen, war der Anteil von Alleinunfällen an allen schweren N3-Unfällen doppelt so hoch wie im Bundesdurchschnitt 2014. Zu Tode kam dabei kein N3-Fahrzeuginsasse, jedoch wurden 22 schwer verletzt. Damit bestätigt sich, dass Alleinunfälle von N3-Güterkraftfahrzeugen zwar selten einen tödlichen Ausgang nehmen, aber für einen erheblichen Anteil - in Brandenburg die Hälfte - der schwer verletzten N3-Insassen ursächlich sind. Bei jedem zweiten Alleinunfall lagen Anzeichen für Müdigkeit beziehungsweise Einschlafen des Fahrers vor; in den anderen Fällen gab es Hinweise auf eine plötzliche Gesundheitsstörung oder einen technischen Defekt am Fahrzeug, meist einen Reifenplatzer.

Eine nähere Betrachtung der 77 Unfälle im Längsverkehr, die somit über die Hälfte der 145 schweren Unfälle im Jahr 2016 ausmachen, zeigt, dass darunter tatsächlich in 25 Fällen ein N3-Güterkraftfahrzeug auf ein vorausfahrendes oder wartendes Fahrzeug auffuhr. In zwei Drittel dieser Fälle geschah dies auf Autobahnen. Soweit es ermittelbar war, stand die Hälfte der Unfallgegner, als der N3-Lkw aufprallte, und die Hälfte der Fahrzeuge fuhr ohne Bremsung auf. Bei Auffahrunfällen durch einen N3Lkw - in zwölf Fällen auf das Heck eines anderen schweren Güterkraftfahrzeugs - wurden sieben Menschen getötet, darunter vier der auffahrenden Lkw-Fahrer. Damit bestätigt sich, dass für die Führer schwerer Güterkraftfahrzeuge das Auffahren auf andere schwere Nutzfahrzeuge die größte Gefahr für das eigene Leben bedeutet, weil es infolge der hohen Massen beider Kollisionsgegner oft zu massiven Verformungen und Eindringungen am Fahrerhaus des Auffahrenden kommt.

Noch häufiger als das Auffahren durch ein N3-Fahrzeug trat allerdings der umkehrte Fall auf: ein Auffahren eines anderen Verkehrsteilnehmers auf das Heck eines N3-Güterkraftfahrzeugs beziehungsweise seines Anhängers. In 35 solcher Unfälle starben drei Menschen im auffahrenden Pkw oder Kleintransporter, 44 wurden schwer verletzt. Mit einer Ausnahme geschahen diese Unfälle auf Autobahnen, wobei der schwere Lkw in den meisten Fällen mit normaler Fahrgeschwindigkeit zwischen 80 und $90 \mathrm{~km} / \mathrm{h}$ vorausfuhr, als der Gegner gegen das Heck prallte. Meist war der Auffahrende auf derselben Fahrspur wie der Lkw unterwegs; in anderen Fällen hatte er allerdings zuvor ein drittes Fahrzeug überholt und war dann hinter dem N3-Güterkraftfahrzeug eingeschert, bevor es zur Kollision kam. Dies legt die Vermutung nahe, dass mitunter die Differenz zwischen der eigenen, deutlich höheren Geschwindigkeit und der des vorausfahrenden Güterkraftfahrzeugs unterschätzt wird, wenn Pkw oder Kleintransporter auf freier Strecke auffahren. Der Heckaufprall führte in den meisten Fällen zum Versagen des gesetzlich vorgeschriebenen Heckunterfahrschutzes am Lkw oder seinem Anhänger, so dass es teilweise zu katastrophalen Deformationen der Fahrgastzelle beim Auffahrenden kam. Die Unfallforschung der Versicherer hat das in einem Versuch, bei dem ein moderner Pkw mit typischer Teilüberdeckung auf das Heck eines stehenden Sattelaufliegers prallte, demonstriert. Obwohl der Unterfahrschutz der aktuellen Richtlinie 2006/20/EG entsprach, riss dieser beim Anprall mit $70 \mathrm{~km} / \mathrm{h}$ ab und führte zu Intrusionen am Pkw, die einem im Aufprallbereich befindlichen Insassen keinen Überlebensraum mehr lassen (Abb. 10).

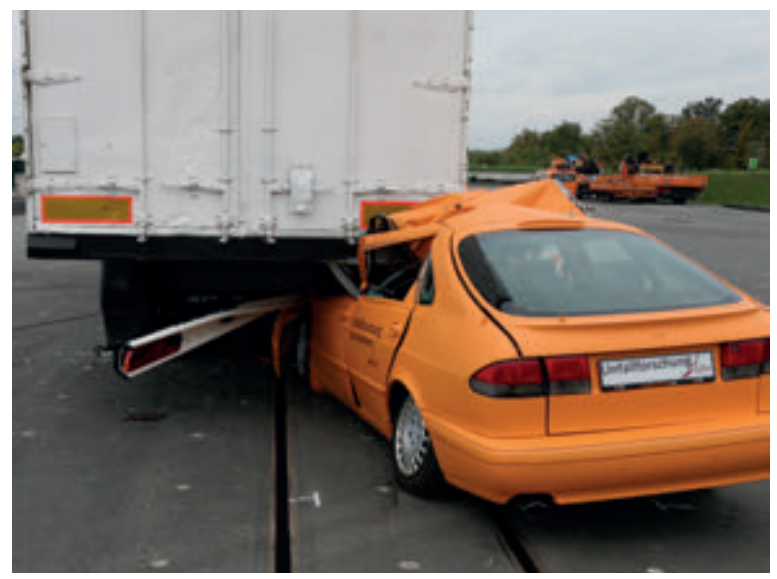

Abb. 10: Abgerissener Heckunterfahrschutz und Deformation am Pkw nach Crashversuch mit $70 \mathrm{~km} / \mathrm{h}$ und Teilüberdeckung 
In den 145 schweren Unfällen in Brandenburg mit Beteiligung von N3-Güterkraftfahrzeugen waren deren Fahrzeugführer zu 48 Prozent die Hauptverursacher. Von diesen wiederum waren gut die Hälfte (56 Prozent) Fahrzeuge mit deutscher Zulassung. Beschränkt man die Betrachtung auf die 76 Unfälle auf Autobahnen, so waren N3-Fahrzeugführer nur in 43 Prozent die Hauptverursacher, von diesen wiederum 58 Prozent Fahrer von ausländischen Lkw. Dieses vermeintliche Übergewicht ausländischer Güterkraftfahrzeuge im Unfallgeschehen relativiert sich jedoch angesichts der Tatsache, dass bei Verkehrszählungen im Rahmen des Forschungsprojekts 57 Prozent ausländische N3-Lkw festgestellt wurden. Deren Unfallbeteiligung spiegelt somit offenbar nur ihren Anteil am Schwerverkehr auf Brandenburgs Autobahnen wider.

\section{Schwere Lkw-Unfälle in der Unfalldatenbank der Versicherer}

Um tiefere Erkenntnisse über typische Umstände und Hergänge schwerer Unfälle mit N3-Güterkraftfahrzeugen zu gewinnen, wurden ergänzend 339 Unfälle mit Beteiligung solcher Lkw aus der Unfalldatenbank der Versicherer im Detail analysiert. Sie basieren auf Kraftfahrthaftpflichtschäden der versicherten Lkw mit einem Schadenaufwand von mindestens 30.000 Euro. Sie sind damit nicht repräsentativ für das allgemeine Unfallgeschehen, sondern legen - wie für das Projektziel gewünscht - den Schwerpunkt auf Fälle, die vom Lkw beziehungsweise dessen Fahrer verursacht wurden, und hohen Schaden und somit oft auch schweren Personenschaden zur Folge hatten.

Auffahrunfälle durch schwere Lkw

Auch in diesem Material nehmen mit 75 Ereignissen Auffahrunfälle durch N3-Güterkraftfahrzeuge, davon 49 auf Autobahnen, erheblichen Raum ein. In 24 Fällen konnten die Fahr- und Kollisionsgeschwindigkeiten des auffahrenden Lkw und zum Teil auch des Unfallgegners bestimmt werden. Die Daten belegen, dass einerseits die Hälfte der Lkw mit Geschwindigkeiten von 80 km/h, teilweise auch darüber, auffuhren und somit keinerlei Bremsung vor der Kollision mehr eingeleitet wurde. Andererseits stand der allergrößte Teil der Unfallgegner zum Zeitpunkt der Kollision (Abb. 11). Die Frage, ob die auffahrenden Lkw bereits mit automatischen Notbremsassistenten ausgerüstet und diese zum Zeitpunkt des Unfalls auch aktiviert waren, lässt sich allerdings nicht beantworten. 
Bekannte Fahr- und Kollisionsgeschwindigkeiten der Unfallbeteiligten o1 und 02 in von Lkw-Fahrern verursachten Auffahrunfällen

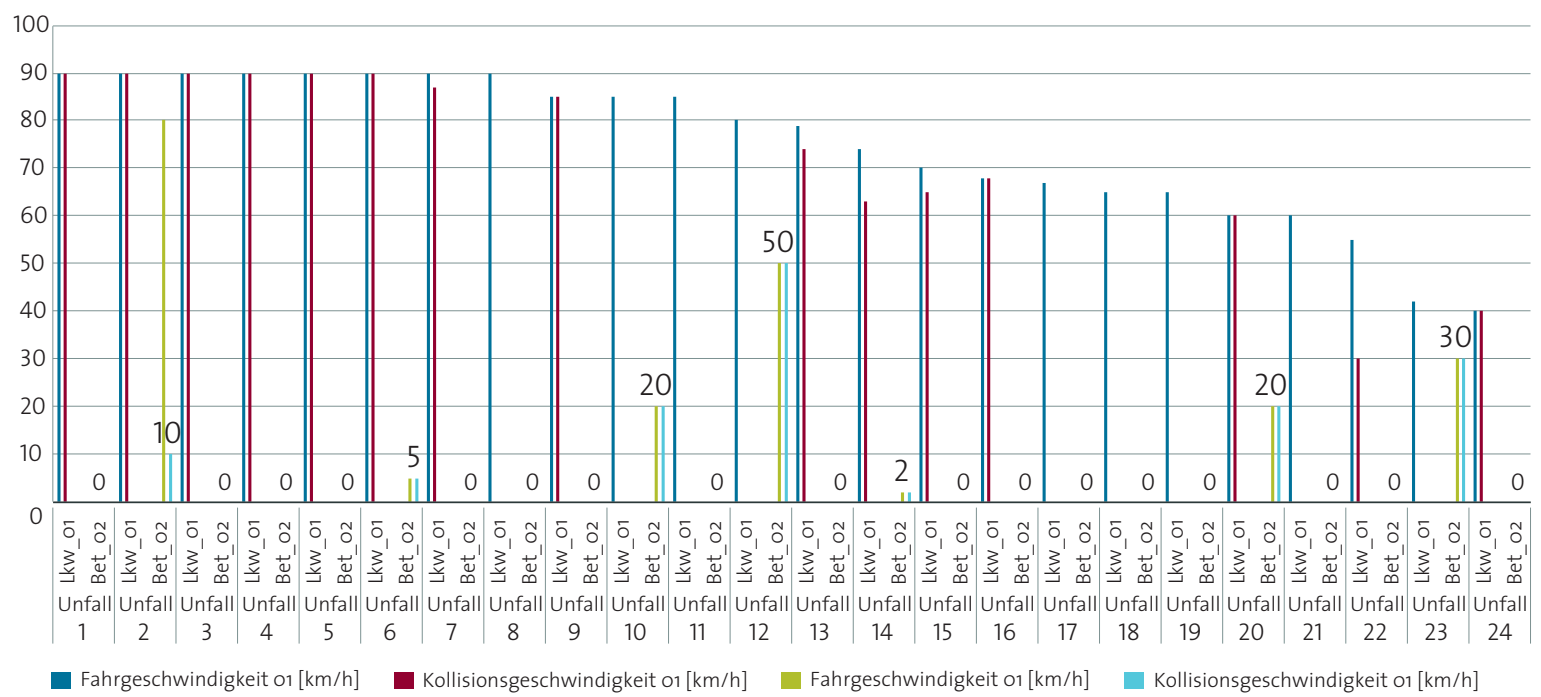

(c) UDV 2018

Abb. 11: Bekannte Fahr- und Kollisionsgeschwindigkeiten des auffahrenden N3-Güterkraftfahrzeugs (Beteiligter 01) und des aufgefahrenen Fahrzeugs (Beteiligter 02) in 24 von Lkw-Fahrern verursachten Auffahrunfällen

Unfälle durch Spurwechsel schwerer Lkw

Unfälle beim Spurwechsel eines N3-Fahrzeugs fanden sich in 35 Fällen im Datenmaterial, davon 24 auf Autobahnen. Sie entsprechen in der Regel dem Unfalltyp „Längsverkehr“ und der Unfallart „Zusammenstoß mit anderem Fahrzeug, das in gleicher Richtung fährt". Zu etwa 60 Prozent führte das N3-Güterkraftfahrzeug dabei einen Spurwechsel nach rechts aus, beispielsweise, um nach einem Überholmanöver auf der Autobahn wieder auf die rechte Fahrspur zu gelangen. Oftmals wurde dann ein rechts neben dem Lkw fahrender Pkw übersehen und angestoßen. In der Regel gingen solche Unfälle zwar mit erheblichem Sachschaden einher, jedoch selten mit schweren Verletzungen. Anders hingegen verhielt es sich bei Fahrspurwechseln von Lkw nach links (40\%), wenn diese auf Autobahnen vollzogen wurden. Dabei kam es in mehreren Fällen zum Zusammenstoß mit einem von hinten auf der Überholspur herannahenden Fahrzeug, welches dann mit erheblicher Differenzgeschwindigkeit gegen die Seite oder das Heck des N3Fahrzeugs prallte.

Abbiege- und Einbiegeunfälle schwerer Lkw

Mit jeweils 40 Fällen stellen Zusammenstöße mit anderen Verkehrsteilnehmern, in denen das N3-Fahrzeug nach links beziehungsweise nach rechts abbiegt oder einbiegt, das zahlenmäßig bedeutendste Szenario im Material der Unfalldatenbank. Beim Abbiegen nach links 

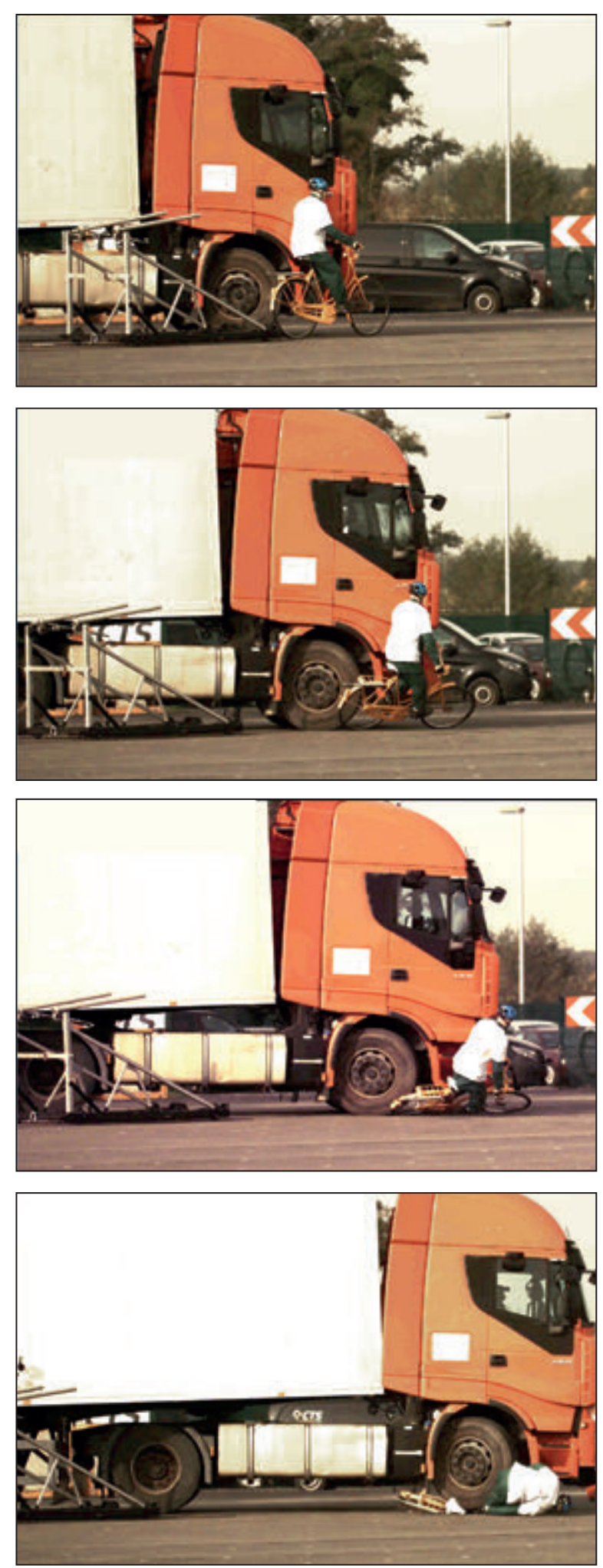

entsteht der Konflikt häufig dadurch, dass ein entgegenkommendes Fahrzeug auf der Gegenfahrbahn übersehen oder die verfügbare Zeitlücke zum Queren der Gegenfahrbahn falsch eingeschätzt wird, so dass es zu einer Frontal-oder Seitenkollision zwischen dem N3-Güterkraftfahrzeug und dem Unfallgegner kommt. Derartige Unfallabläufe sind zwar auch von Pkw her bekannt. Jedoch sind schwere Lkw aufgrund ihrer Masse und Steifigkeit für alle anderen Verkehrsteilnehmer ungleich stärkere Kontrahenten, weshalb sich unter den 30 Kollisionen mit Schwerverletzten und Getöteten dieser Konstellation auch neun Pkw und zwölf motorisierte Zweiräder fanden.

Unfälle mit nach rechts abbiegenden oder einbiegenden N3-Güterkraftfahrzeugen wurden mit 70 Prozent durch Kollisionen mit Radfahrern dominiert. Von zwei Ausnahmen abgesehen bewegten sich diese zunächst in gleicher Richtung wie der Lkw und wurden dann im Abbiegevorgang mit der rechten Seite des Fahrzeugs oder rechten vorderen Ecke des Fahrerhauses umgestoßen und oftmals im Anschluss überrollt. Abb. 12 demonstriert den typischen Ablauf im Crashversuch. Zusammenstöße mit ungeschützten Verkehrsteilnehmern beim Rechtsabbiegen blieben in vielen Fällen vom Lkw-Fahrer zunächst unbemerkt, was erklärt, dass die Opfer mitunter von mehreren Achsen überrollt wurden. Ob die Ursache dafür letztlich in mangelnder Aufmerksamkeit des Fahrers oder schlechter Erkennbarkeit des ungeschützten Verkehrsteilnehmers lag, ließ sich nicht ermitteln. In den allermeisten Fällen befanden sich die Radfahrer aber über einen Zeitraum von vermutlich mehreren Sekunden neben dem Lkw und be-

Abb. 12: Typischer Ablauf eines Rechts-Abbiege-Unfalls, bei dem sich der Radfahrer einige Zeit neben dem Fahrerhaus des Lkw bewegt und beim Abbiegen mit der rechten vorderen Ecke des Fahrerhauses umgestoßen und mit dem Vorderrad überrollt wird 
wegten sich nur mit mäßiger Geschwindigkeit, bevor es zur Kollision kam. Mit vier Getöteten und 23 Schwerverletzten repräsentierte diese Gruppe auch den größten Anteil bei den schweren Unfallfolgen. Mit durchschnittlich 53 Jahren waren eher ältere Radfahrer in Kollisionen mit Lkw verwickelt, in einigen Fällen aber auch Jugendliche und Kinder. In Übereinstimmung mit der Sonderauswertung der Bundesstatistik ereigneten sich die Unfälle im Material der Unfalldatenbank innerorts, in der Regel bei Tageslicht und trockenen Witterungsverhältnissen und oftmals mit Lkw ohne Anhänger. Anhand ihrer Aufbauten waren gut 40 Prozent der Fahrzeuge der Bau- und Entsorgungswirtschaft zuzuordnen.

In Ergänzung zu den drei Unfallanalysen als Säulen des Forschungsprojektes wurden weitere Untersuchungen auf und an Autobahnen, vorrangig in Brandenburg, durchgeführt. Es wurden sowohl Befragungen von Lkw-Fahrern zu den Arbeitsbedingungen und der Akzeptanz von Fahrerassistenzsystemen durchgeführt als auch Lkw-Fahrerbeobachtungen und Lkw-Geschwindigkeitsmessungen im fließenden Verkehr vorgenommen. 
Fazit und Empfehlungen

\section{Fazit und Empfehlungen}

Bei Unfällen mit Beteiligung schwerer Lkw (EU-Typklasse $\mathrm{N}_{3}$, das heißt über 12 Tonnen zulässiges Gesamtgewicht) sterben pro Jahr fast 500 Menschen und etwa 3.200 werden schwer verletzt. Wenngleich Führer von N3-Fahrzeugen seltener Hauptverursacher von Unfällen mit Personenschaden sind als Fahrer von Pkw oder leichteren Güterkraftfahrzeugen, so kommt es aufgrund der hohen Massen und steifen Strukturen dieser Fahrzeuge häufiger zu schweren Unfallfolgen (Getötete und Schwerverletzte). Mit Maßnahmen zur Verbesserung der passiven Sicherheit, wie der Einführung des Frontunterfahrschutzes, hat es in der Vergangenheit Fortschritte gegeben. Ausnahmen von gesetzlichen Regelungen, wie der Entfall des Front- und seitlichen Unterfahrschutzes an geländegängigen Lkw, müssen dabei auf ein Minimum begrenzt werden. Andere Anforderungen, wie die Erhöhung der Festigkeit des Heckunterfahrschutzes, müssen verschärft werden, weil sie den im realen Unfallgeschehen auftretenden Anprallbelastungen selten standhalten.

Besondere Aufmerksamkeit muss jedoch der Verbreitung und Verbesserung von Fahrerassistenzsystemen für Lkw gelten. Die seit kurzem gesetzlich geforderte obligatorische Ausstattung mit einem automatischen Notbremssystem (AEBS) und einem Spurverlassenwarner (LDWS) weist den Weg in die richtige Richtung, ist in ihren technischen Leistungsanforderungen und mit ihren Ausnahmen aber nicht befriedigend. Im Rahmen des Forschungsprojektes wurde festgestellt, dass ein Großteil der Auffahrunfälle von Lkw auf Autobahnen auf stehende Fahrzeuge am Stauende und - konform mit den Ergebnissen der durchgeführten Geschwindigkeitsmessungen - aus einer Geschwindigkeit des Lkw von etwa 90 km/h geschieht. Die gesetzlich geforderte Abbremsung eines Lkw mit AEBS aus einer Geschwindigkeit von $80 \mathrm{~km} / \mathrm{h}$ um $20 \mathrm{~km} / \mathrm{h}$ auf ein stehendes Fahrzeug ist daher als unzureichend anzusehen. Fahrzeuge ohne hintere Luftfederung - dazu gehören in der Regel schwere Baufahrzeuge - sind von der Ausstattungspflicht gänzlich ausgenommen. Die Ergebnisse der Lkw-Fahrer-Be-

fragung weisen darauf hin, dass diese und andere Fahrerassistenzsysteme von den Fahrern in der großen Mehrheit akzeptiert und selten abgeschaltet werden. Mit weiterer Erhöhung der Zuverlässigkeit der Fahrerassistenzsysteme sollte deren Abschaltbarkeit zukünftig deutlich erschwert werden, um ihr Schutzpotenzial auszuschöpfen. Eine dauerhafte Deaktivierung nach Fahrtantritt muss ausgeschlossen sein.

Unfälle zwischen abbiegenden Lkw und ungeschützten Verkehrsteilnehmern, insbesondere Radfahrern, sind besonders häufig durch schwerste und tödliche Folgen gekennzeichnet. Im vorliegenden Material der Unfalldatenbank der Versicherer waren etwa zwei Drittel der Unfälle zwischen schweren Lkw und Radfahrern auf eine RechtsAbbiege-Situation zurückzuführen. Da Kollisionen mit Radfahrern oftmals durch den Lkw-Fahrer anfänglich unbemerkt bleiben, wird einem elektronischen Rechts-Abbiege-Assistenten, der den Raum rechts neben dem Fahrzeug überwacht und bei Detektion eines Radfahrers oder Fußgängers eine Warnung ausgibt, ein hohes Unfallvermeidungspotenzial zugeschrieben. Ein Lkw-Hersteller bietet seit Ende 2016 einen radarbasierten Rechts-Abbiege-Assistenten an, derzeit allerdings nur für Sattelzugmaschinen und dreiachsige Lkw im Straßengüterverkehr. Die Unfallanalysen zeigen jedoch, dass etwa 40 Prozent der an solchen Unfällen beteiligten Lkw Fahrzeuge der Bau- und Entsorgungsbranche sind. 


\section{Unfallforschung der Versicherer

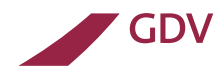

Gesamtverband der Deutschen

Versicherungswirtschaft e.V.

Wilhelmstraße 43/43 G, 10117 Berlin

Postfach 0802 64, 10002 Berlin

Telefon $030.2020-5821$

Fax $030.2020-6633$

unfallforschung@gdv.de

www.udv.de

www.gdv.de

Facebook: facebook.com/unfallforschung

Twitter: @unfallforschung

YouTube: youtube.com/unfallforschung

Instagram: instagram.com/udv_unfallforschung

Redaktion:

Dr.-Ing. Axel Malczyk

Gestaltung:

pensiero KG, wWw.pensiero.eu

Bildquellen:

Die Nutzungsrechte der in dieser

Broschüre abgebildeten Fotos liegen bei der Unfallforschung der Versicherer.

Erschienen: 08/2018 


\section{GDV}

DIE DEUTSCHEN VERSICHERER

Gesamtverband der Deutschen Versicherungswirtschaft e.V.

Wilhelmstraße 43 / 43G, 10117 Berlin

Postfach 080264,10002 Berlin

Tel.: 030/20 $20-50$ oo, Fax: 030/2020-6000

www.gdv.de, www.udv.de 